\section{JTI}

JOURNAL OF

TRAUMA AND INJURY

Received: September 7, 2020

Accepted: September 17, 2020

\section{Correspondence to}

Ye Rim Chang, M.D., Ph.D.

Department of Trauma Surgery, Trauma Center, Dankook University Hospital, 201

Manghyang-ro, Dongnam-gu, Cheonan 31116, Korea

Tel: +82-41-550-3066

Fax: +82-41-550-0039

E-mail:yrchang@dkuh.co.kr

\title{
Effects of Resuscitative Endovascular Balloon Occlusion of the Aorta in Neurotrauma: Three Cases
}

\author{
Dong Hun Kim, M.D., M.S. , Ye Rim Chang, M.D., Ph.D. , \\ Jung-Ho Yun, M.D., M.S. ${ }^{2}$ \\ ${ }^{1}$ Department of Trauma Surgery, Trauma Center, Dankook University Hospital, Cheonan, \\ Korea \\ ${ }^{2}$ Department of Neurosurgery, Trauma Center, Dankook University Hospital, Cheonan, \\ Korea
}

\begin{abstract}
Resuscitative endovascular balloon occlusion of the aorta (REBOA) is widely performed as an adjunct to resuscitation or bridge to definitive control of non-compressible torso hemorrhage in patients with hemorrhagic shock. It is a crucial adjunct for the maintenance of cerebral and coronary perfusion during resuscitation. However, in polytrauma patients with concomitant neurotrauma, such as traumatic brain injury (TBI) or spinal cord injury, the physiological effects of REBOA are unclear. In this report on REBOA performed in a clinical setting for polytrauma patients with spinal cord injury or TBI, the physiological effects of REBOA in neurotrauma are reviewed.
\end{abstract}

Keywords: Trauma; Spinal cord; Brain; Balloon occlusion; Resuscitation

\section{INTRODUCTION}

Resuscitative endovascular balloon occlusion of the aorta (REBOA) is performed as a bridging therapy for non-compressible torso hemorrhage (NCTH) in patients with hemorrhagic shock [1]. It is also performed for patients with neurogenic shock and non-traumatic cardiac arrest to support the central aortic pressure necessary for cerebral and coronary perfusion [2,3]. However, when hemorrhagic shock occurs in patients with concomitant traumatic brain injury (TBI) or spinal cord injury (SCI), the effect of REBOA on the concomitant neurotrauma is unclear. In this report, we present three patients, including one with cervical SCI and NCTH and 2 with TBI and $\mathrm{NCTH}$, who underwent REBOA, and review the effects of REBOA on the physiology (http://creativecommons.org/licenses/by-nc/4.0/) which permits unrestricted noncommercial use, distribution, and reproduction in any medium, provided the original work is properly cited. 
of neurotrauma.

\section{CASE REPORT}

\section{Case 1}

A 67-year-old woman had a traffic accident, and cardiac arrest was noted at the accident scene and at a local hospital with return of spontaneous circulation (ROSC) after 7 and 14 minutes of cardiopulmonary resuscitation (CPR), respectively. Upon arrival from the local hospital, she was comatose and intubated, her pupils were $4 \mathrm{~mm}$ in size and fixed, and her vital signs were as follows: blood pressure, 79/64 mmHg; pulse rate, $128 \mathrm{bpm}$; and body temperature, $36.0^{\circ} \mathrm{C}$. Focused Assessment with Sonography for Trauma (FAST) did not show the lung sliding sign in the right hemithorax or intra-abdominal fluid collection. A lateral-view radiograph of the cervical spine revealed suspected atlanto-occipital dislocation (AOD) (Fig. 1A). Computed tomography (CT) at the local hospital showed grade I spleen contusion, retroperitoneal hematoma around the left kidney, multiple rib fractures with hemopneumothorax in the right hemithorax, and AOD with a 25-mm basion-dental interval (BDI) and prevertebral hematoma (Fig. 1B). Despite volume resuscitation with crystalloid and blood transfusions, hypotension with a systolic blood pressure (SBP) less than $70 \mathrm{mmHg}$ persisted. Therefore, Zone I REBOA using a 7-Fr balloon cathe- ter (RESCUE Balloon ${ }^{\text {TM }}$, Tokai Medical Products, Aichi, Japan) was performed (Fig. 1C), and her blood pressure increased temporarily and then decreased despite $40-\mathrm{mL}$ full balloon inflation and high-dose vasopressor infusion. Cardiac arrest recurred 8 minutes after balloon inflation, and ROSC was not achieved in 36 minutes of CPR.

\section{Case 2}

A 58-year-old man sustained blunt trauma from an 8-m fall. His Glasgow Coma Scale score was 3. His pupils had a size of $6 \mathrm{~mm}$ on light reflex and were fixed. Upon arrival, his blood pressure and pulse rate were $98 / 60 \mathrm{mmHg}$ and $92 \mathrm{bpm}$ respectively, and he had multiple facial abrasions. FAST showed no intraabdominal fluid collection, but 20-minute serial FAST showed a small amount of fluid in the Morison pouch and perisplenic space. A CT scan revealed subdural hematoma $(\mathrm{SDH})$ in the right hemisphere with a vague boundary between white and gray matter consistent with brain edema (Fig. 2A), multiple rib fractures, and central inframesocolic retroperitoneal hematoma in the para-aortic area beneath the left renal vein with small fluid collection in the abdominal cavity (Fig. 2B). After the CT scan, the patient became hemodynamically unstable with a blood pressure of $61 / 38 \mathrm{mmHg}$ and a pulse rate of $91 \mathrm{bpm}$. Zone I REBOA was then performed using a RESCUE Balloon ${ }^{\mathrm{TM}}$ (Fig. 2C). Following balloon inflation with $15 \mathrm{~mL}$ of saline, his blood pressure and pulse rate increased to $101 / 60 \mathrm{mmHg}$ and $112 \mathrm{bpm}$,
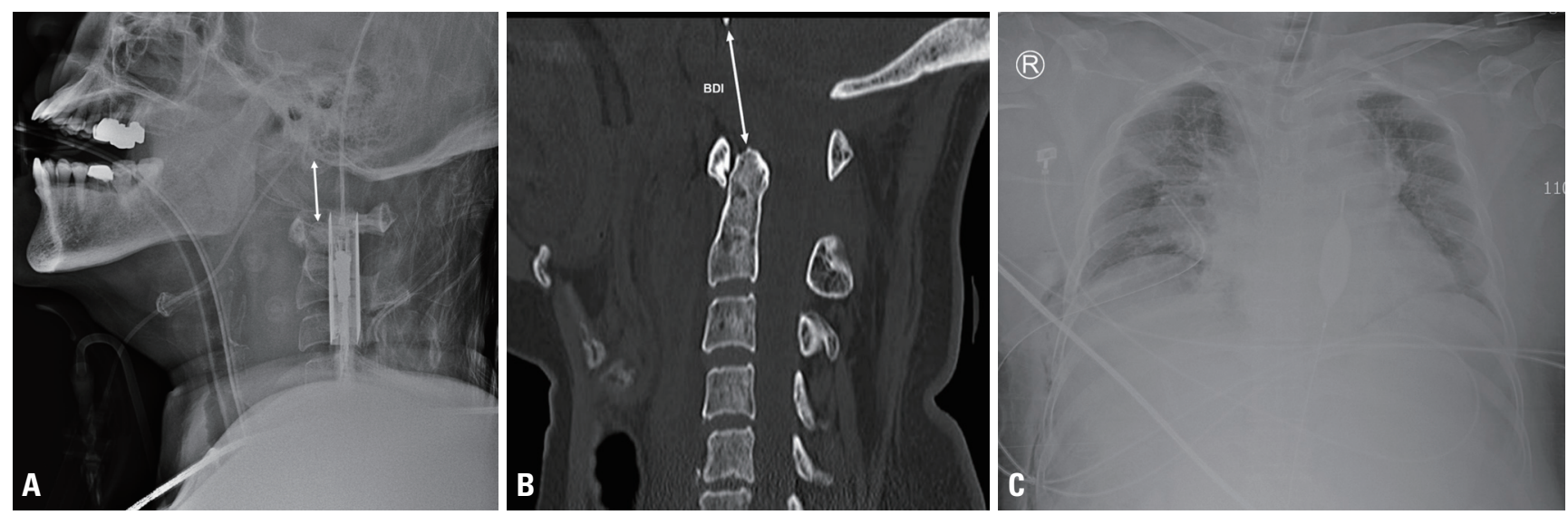

Fig. 1. (A) Lateral-view radiograph of the cervical spine showing a distraction-type AOD. (B) Computed tomography scan showing AOD with a 25-mm basion-dental interval and a thick prevertebral hematoma. (C) Chest radiograph showing the positioning of the balloon catheter in the descending thoracic aorta. AOD: atlanto-occipital dislocation, BDI: basion-dental interval. 
respectively. Simultaneous craniectomy and laparotomy were then performed. A central inframesocolic retroperitoneal hematoma and superficial liver and spleen lacerations with a small intraperitoneal hematoma were found during crash laparotomy. As the retroperitoneal hematoma was approached and controlled by midline infrarenal aortic exposure, it was possible to complete weaning from REBOA. However, the bleeding from the liver and spleen lacerations were not easily controlled even though the injuries were minor. During laparotomy, the SDH was evacuated and severe brain edema was observed by craniectomy. Since the brain injury was virtually irreversible, both surgical procedures were finished without further steps, and laparotomy was finished with temporary abdominal closure. Postoperatively, the patient remained hemodynamically unstable despite volume resuscitation and high-dose vasopressor infusion, and he died $341 \mathrm{~min}$ utes after arrival.

\section{Case 3}

A 72-year-old man was admitted after a motorcycle accident. Upon arrival, he was comatose, and his pupils were $3 \mathrm{~mm}$ in size and fixed. He had a blood pressure of 71/54 $\mathrm{mmHg}$, a pulse rate of $120 \mathrm{bpm}$, a respiratory rate of 12 breaths/minutes, and a hemoglobin level of $9.5 \mathrm{~g} / \mathrm{dL}$. He had multiple facial lacerations and cerebrospinal fluid
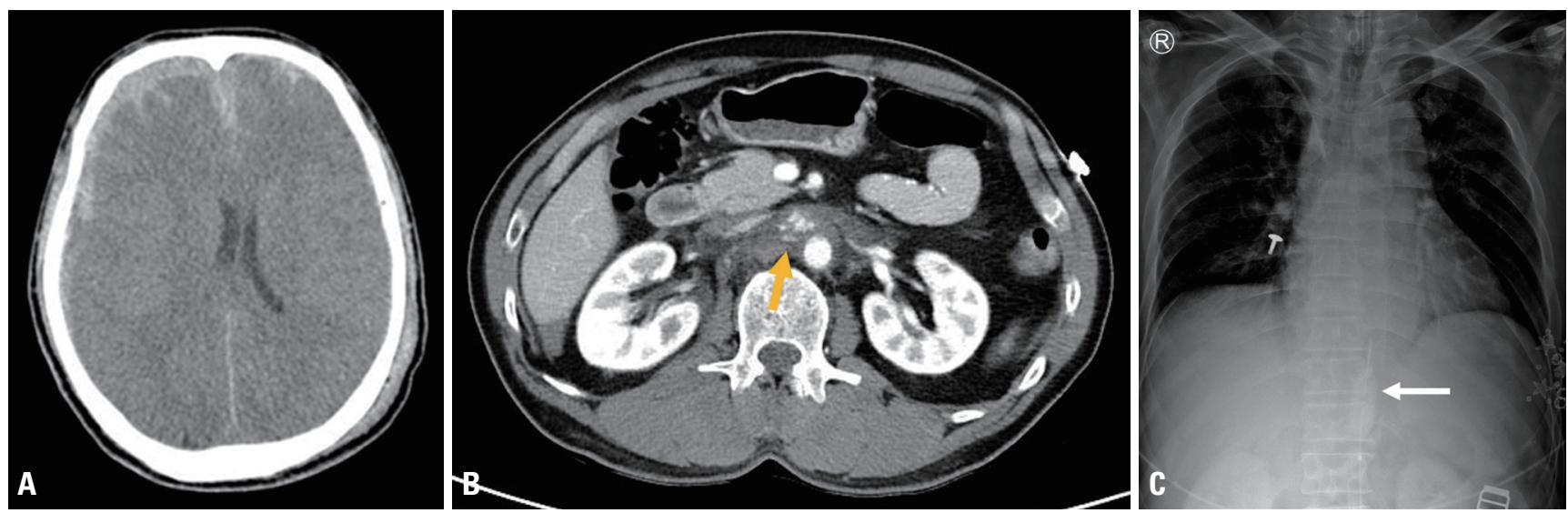

Fig. 2. (A) Brain computed tomography (CT) scan showing subdural hemorrhage and subarachnoid hemorrhage in the right hemisphere with midline shifting and diffuse brain edema. (B) Abdominal CT scan showing para-aortic hematoma with contrast extravasation beneath the left renal vein (arrow) and fluid collection in the abdominal cavity. (C) Chest radiograph showing that the positioning of the balloon catheter (arrow) had been checked in the abdominal aorta, and the catheter was then adjusted $7 \mathrm{~cm}$ in the cranial direction for proper positioning in the thoracic aorta in Zone I.
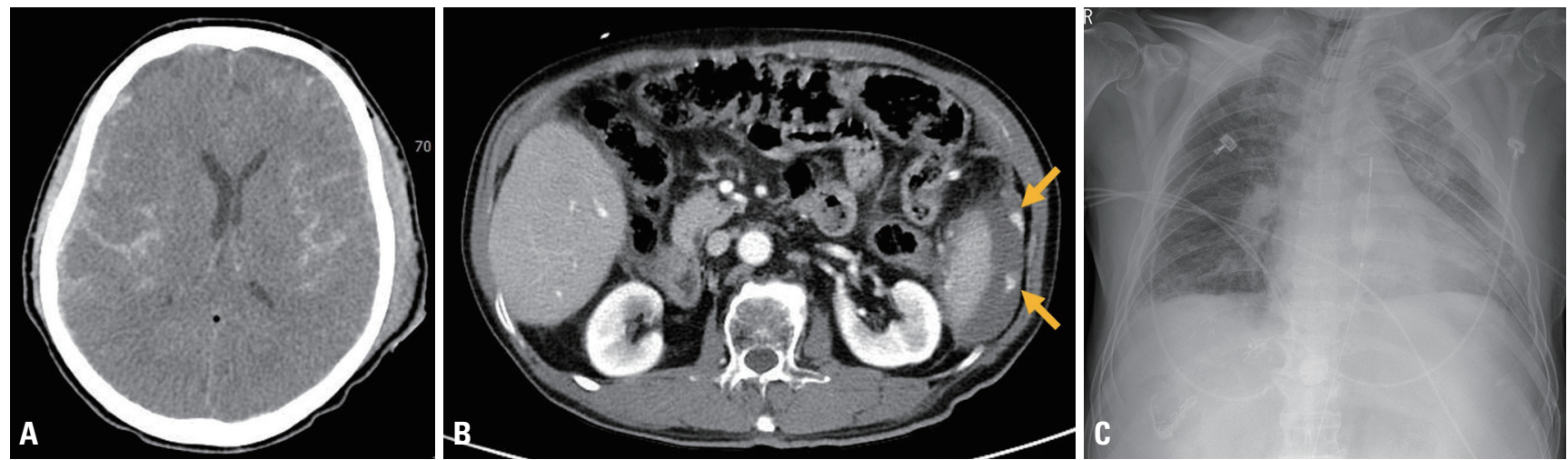

Fig. 3. (A) Brain computed tomography (CT) scan showing subdural hemorrhage and subarachnoid hemorrhage in both hemispheres with diffuse brain edema. (B) Abdominal CT scan showing spleen laceration with contrast extravasation (arrows) and fluid collection in the perisplenic space. (C) Chest radiograph showing proper positioning of the balloon catheter in the descending thoracic aorta. 
leakage from the nose and left ear. FAST did not initially show intra-abdominal fluid collection, but 10-minute serial FAST showed a small amount of fluid in the splenorenal recess. Since the patient responded to volume resuscitation, he could undergo imaging evaluation. A CT scan revealed subarachnoid hemorrhage and SDH in both hemispheres with basal skull fracture and pneumocephalus (Fig. 3A), a grade III spleen laceration with contrast extravasation and perisplenic fluid collection (Fig. 3B), and multiple rib fractures with hemopneumothorax in the left hemithorax. Shortly after the imaging evaluation, hypotension gradually developed even though volume resuscitation and low-dose vasopressor infusion were continued. With his blood pressure at 48/26 $\mathrm{mmHg}$ and pulse rate at $89 \mathrm{bpm}$, Zone I REBOA using a RESCUE Balloon ${ }^{\mathrm{TM}}$ was performed 55 minutes after arrival (Fig. 3C). After 11-mL balloon inflation, his blood pressure and pulse rate rose to $127 / 76 \mathrm{mmHg}$ and $128 \mathrm{bpm}$, respectively. Since a poor outcome was expected due to severe brain edema, craniectomy was performed prior to laparotomy. Craniectomy revealed severe brain edema after hematoma removal and was completed with suspension of the crash laparotomy. Weaning from REBOA was completed in the

Table 1. Critical time courses and clinical features of patients with regards to REBOA

\begin{tabular}{|lccc|}
\hline Parameters & Case 1 & Case 2 & Case 3 \\
\hline Door-to-femoral puncture time (minutes) & 33 & 84 & 55 \\
\hline Door-to-balloon inflation time (minutes) & 40 & 91 & 66 \\
\hline Maximal volume of balloon inflation (mL) & 40 & 15 & 20 \\
\hline SBP before balloon inflation (mmHg) & 62 & 61 & 48 \\
\hline SBP after balloon inflation ( $\mathrm{mmHg})$ & 66 & 105 & 127 \\
Maximal SBP during REBOA (mmHg) & 66 & 109 & 140 \\
Total duration of aortic occlusion (minutes) & 40 & 125 & 138 \\
Hemoglobin level on arrival (g/dL) & 7.8 & 14.3 & 10.7 \\
\hline Lactate level on arrival (mmol/L) & 8.6 & 3.3 & 3.6 \\
INR on arrival & 1.70 & 1.17 & 1.26 \\
24-hour transfusion units required (RBC:FFP) & $8: 5$ & $15: 10$ & $21: 15$ \\
\hline Door-to-operating room time (minutes) & - & 106 & 82 \\
\hline Door-to-death time (minutes) & 90 & 341 & 396 \\
\hline
\end{tabular}

FFP: fresh frozen plasma, INR: international normalized ratio, RBC: red blood cells, REBOA: resuscitative endovascular balloon occlusion of the aorta, SBP: systolic blood pressure. intensive care unit about 1 hour after the operation and replaced with vasopressor infusion. The patient remained hemodynamically unstable despite high-dose vasopressor infusion and died 4 hours after the operation.

The injury severity scores of patients 1,2, and 3 were 50 , 38 , and 43 , respectively. The procedural timepoints with regards to REBOA and the clinical features of the three patients are shown in Table 1.

\section{DISCUSSION}

REBOA is a bridging modality for patients with hemorrhagic shock that is aimed at maintaining cerebral and coronary perfusion, while reducing distal bleeding, until hemorrhage is controlled. It is widely performed in cases of postpartum hemorrhage, upper gastrointestinal bleeding, pelvic surgery, trauma, ruptured aortic aneurysm, and non-traumatic cardiac arrest [2,4]. The main indication for REBOA in trauma settings is NCTH with hemorrhagic shock including abdominal or pelvic hemorrhage [1]. However, traumatic accidents often involve complex injury patterns. Patients often suffer polytrauma, in which a neurological injury such as TBI or SCI is among the most common factors. NCTH with concomitant traumatic neurological injury is not known to be a contraindication of REBOA. Thus, it is important to understand the effects of REBOA on NCTH in patients with hemorrhagic shock and concomitant neurological injury.

There are few reports and no consensus on REBOA as an adjunct for patients with neurogenic shock resulting from traumatic spine injury. Gray and Dieudonne [3] reported a case of successful REBOA in a patient with neurogenic shock secondary to a comminuted fracture at C5. In case 1, high-level SCI secondary to AOD might have resulted in brainstem injury unresponsive to complete occlusion of aortic flow, which is termed complete REBOA. A BDI $\geq 16 \mathrm{~mm}$ or AOD with a neurological deficit is especially associated with mortality [5]. Further, in an animal study on Zone I REBOA using a primate model, it was reported that a long duration of balloon inflation during REBOA may result in spinal cord ischemia [6]. Therefore, we should keep in mind that patients with traumatic SCI may be more vulnerable to ischemic and 
reperfusion injury from REBOA.

Hemorrhagic shock in trauma patients often occurs with concomitant TBI; therefore, it is important to understand the relationship between aortic occlusion and cerebral physiology following TBI to maintain optimal physiology of the vital organs. Aortic occlusion increases coronary and cerebral perfusion [7]. According to a reported smooth U-shaped correlation between SBP and TBI outcomes, outcomes can improve as SBP increases up to $135 \mathrm{mmHg}$ [8]. Thus, supra-physiological blood pressure and carotid blood flow created by aortic occlusion may worsen cerebral edema, increase the intracranial pressure, or exacerbate intracranial hemorrhage [9-11]. Moreover, as Zone I REBOA immediately increases blood pressure substantially more than Zone III REBOA, this may have important implications for cerebral function and perfusion goals, particularly in patients with concomitant TBI [12]. In cases 2 and 3, partial REBOA (pREBOA) in zone I was performed, and resuscitation was successful, but severe brain edema secondary to TBI before REBOA contributed to rapid worsening of shock and created a situation that was difficult to reverse with surgical intervention.

In animal studies, pREBOA performed to allow titrated and controlled low-volume aortic flow distal to the site of occlusion resulted in physiological patterns similar to baseline, with smaller increases in the intracranial pressure and proximal mean arterial pressure, and did not aggravate TBI, although prolonged pREBOA may worsen the severity of shock $[9,13]$.

REBOA for NCTH in patients with concomitant neurotrauma such as TBI and SCI has several challenges, including insufficient clinical and preclinical data and a lack of consensus on simultaneous operations for brain and torso hemorrhage. Extensive studies are needed to determine the optimal use of REBOA in neurotrauma patients with hemorrhagic shock or other shock states.

\section{REFERENCES}

1. Napolitano LM. Resuscitative endovascular balloon occlusion of the aorta: indications, outcomes, and training. Crit Care Clin 2017;33:55-70
2. Daley J, Morrison JJ, Sather J, Hile L. The role of resuscitative endovascular balloon occlusion of the aorta (REBOA) as an adjunct to ACLS in non-traumatic cardiac arrest. Am J Emerg Med 2017;35:731-6.

3. Gray S, Dieudonne B. Resuscitative endovascular balloon occlusion of the aorta as an adjunct in a patient with neurogenic shock. Cureus 2018;10:e3375.

4. Morrison JJ, Galgon RE, Jansen JO, Cannon JW, Rasmussen TE, Eliason JL. A systematic review of the use of resuscitative endovascular balloon occlusion of the aorta in the management of hemorrhagic shock. J Trauma Acute Care Surg 2016;80:324-34.

5. Cooper Z, Gross JA, Lacey JM, Traven N, Mirza SK, Arbabi S. Identifying survivors with traumatic craniocervical dissociation: a retrospective study. J Surg Res 2010;160:3-8.

6. Eliason JL, Myers DD, Ghosh A, Morrison JJ, Mathues AR, Durham L, et al. Resuscitative endovascular balloon occlusion of the aorta (REBOA): zone I balloon occlusion time affects spinal cord injury in the nonhuman primate model. Ann Surg 2019 Jun 7. doi: 10.1097/SLA.0000000000003408 [Epub ahead of print].

7. Bailey ZS, Cardiff K, Yang X, Gilsdorf J, Shear D, Rasmussen TE, et al. The effects of balloon occlusion of the aorta on cerebral blood flow, intracranial pressure, and brain tissue oxygen tension in a rodent model of penetrating ballistic-like brain injury. Front Neurol 2019;10:1309.

8. Butcher I, Maas AI, Lu J, Marmarou A, Murray GD, Mushkudiani NA, et al. Prognostic value of admission blood pressure in traumatic brain injury: results from the IMPACT study. J Neurotrauma 2007;24:294-302.

9. Johnson MA, Williams TK, Ferencz SE, Davidson AJ, Russo RM, O'Brien WT Sr, et al. The effect of resuscitative endovascular balloon occlusion of the aorta, partial aortic occlusion and aggressive blood transfusion on traumatic brain injury in a swine multiple injuries model. J Trauma Acute Care Surg 2017;83:6170.

10. Norii T, Crandall C, Terasaka Y. Survival of severe blunt trauma patients treated with resuscitative endovascular balloon occlusion of the aorta compared with propensity score-adjusted untreated patients. J Trauma Acute Care Surg 2015;78:721-8.

11. Uchino H, Tamura N, Echigoya R, Ikegami T, Fukuoka T. "REBOA" - Is it really safe? A case with massive intracranial hemorrhage possibly due to endovascular balloon occlusion of the aorta (REBOA). Am J Case Rep 2016;17:810-3.

12. Wasicek PJ, Li Y, Yang S, Teeter WA, Scalea TM, Hu P, et al. Ex- 
amination of hemodynamics in patients in hemorrhagic shock undergoing resuscitative endovascular balloon occlusion of the aorta (REBOA). Injury 2019;50:1042-8.

13. Williams AM, Bhatti UF, Dennahy IS, Graham NJ, Nikolian VC,
Chtraklin K, et al. Traumatic brain injury may worsen clinical outcomes after prolonged partial resuscitative endovascular balloon occlusion of the aorta in severe hemorrhagic shock model. J Trauma Acute Care Surg 2019;86:415-23. 